\title{
The Algorithm of Congestion Control Based on Satellite Switching
}

\author{
Zhang Yi, Li Jun, Li Jingling, Zhou Quan, and Liang Wei
}

\begin{abstract}
In order to improve satellite network performance, an algorithm of congestion control based on traffic of Ground Terminals is proposed in this paper. Because the resource in satellite switching system is limited, some different strategies are adopted to control the congestion in this algorithm, which combines open-loop and close-loop based on the current state of onboard buffer. The analysis and simulation results show that the proposed algorithm has the better performance in cell loss rate, and congestion in the onboard buffer can be avoided, which guarantees the switching performance of satellite network.
\end{abstract}

Index Terms-Satellite switching, congestion control, discarding strategy.

\section{INTRODUCTION}

One of the development trends in satellite communication is onboard switching, by which satellite can switch the data among multi-beams. Accordingly, the satellite switching will greatly accelerate the development of satellite communication [1].

On the other hand, the resource of satellite switching system is limited. If the total payload in local time exceeds the maximum throughput of the system, large number cells will be lost and there will been a sharp decline in the performance of system, which is named congestion [2], [3]. Only increasing the buffer of onboard switching can delay the congestion, but the congestion phenomenon is unable to be decreased or resolved. The onboard switching also cannot be self-recovery by this strategy. Consequently, it is necessary to study the congestion control strategy in the satellite switch systems, in order to guarantee the switching performance of satellite network.

With the characteristics of satellite-to-earth links, an algorithm of congestion control based on traffic of ground terminals is proposed in this paper. The algorithm adopts different congestion control strategies, which are based on the current state of onboard buffer, in order to increase the performance of onboard switching.

This paper is arranged as follows: the new congestion control algorithm we propose is described in Section II, in which the idea of the algorithm is introduced in detail; the implementation way and flow are showed in Section III; then

Manuscript received March 5, 2015; revised November 4, 2015. This work was supported by the National Natural Science Foundation of China under Grant No.61372175 and the National Key Laboratory Foundation of China under Grant No.9140C530403130C53192.

The authors are with National Key Laboratory of Science and Technology on Space Microwave, Xi'an 710100, China (e-mail: zhangy4@cast504.com, lij41@cast504.com, lij188@cast504.com, Zhouq97@cast504.com, liangw@cast504.com). the simulation results of the new algorithm is analyzed in Section IV. At last, Section V concludes the paper.

\section{The Algorithm OF CONGESTION CONTROL BASED ON SATELLITE SWITCHING}

The solutions of congestion control are commonly divided two classes. One of the solutions is Open-Loop control, and the other is Close-loop control. The Open-Loop control strategy is a prevention policy, which restricts the traffic of input cell by the prior knowledge or some control measures, in order to reduce the probability of congestion and enable the onboard switching to recover from the severely congestion. On the other hand, the Close-Loop control strategy is a reactive policy. In this strategy, the onboard switching sends control message to ground source terminal in order to reduce the cell sending rate of ground source terminal when the congestion in the onboard switching gets worse.

Because the satellite-to-earth links have long delay, the adopted congestion control strategy is Open-Loop commonly [4], [5]. But the problem of congestion in onboard switching can't be resolved greatly only using this method. When the cell sending rates of ground source terminal exceeds the reservation rate, the total payload in local time may exceed the maximum throughput of the system. Accordingly, we propose an algorithm of congestion control based on traffic of ground terminals, which adopts different congestion control strategies based on the current state of onboard buffer.

In satellite switching system, the ground source terminals should send the connection setup request message to onboard switching before sending data. Then the onboard switching decides whether or not to agree the request according to the available resource in the onboard switching and the characteristic of downlink and uplink. If the connection setup request is agreed by onboard switching, the resource such as reservation bandwidth and connection references value are reserved, then the onboard switching sends message to ground source terminals which indicates the connection setup request message is agreed. The ground source terminals can send data only when receiving the connection setup success message from onboard switching.

When the congestion appears in the onboard switching because of the high traffic burst load or the sending rate of ground source terminal exceeding the reservation rate, the total payload in local time will exceed the maximum throughput of the system. The onboard switching decides whether or not to send alert message to the ground source terminals or discard all the cells of one connect directly according to the congestion level. 


\section{THE REALIZATION OF CONGESTION CONTROL ALGORITHM BASED ON SATELLITE SWITCHING}

\section{A. Steps of Realization}

The congestion control algorithm based on satellite switching proposed in this paper can be realized as follows.

Firstly, the ground source terminals send the connection setup request message to the onboard switching, which conclude parameters of the connection, such as services metric, traffic type, QoS Requirement, and so on. The onboard switching decides whether or not to accept this request by the available resource in the onboard switching and the characteristic of downlink and uplink. If this request is accepted, the onboard switching sends the connection setup success message to the ground source terminal. Then the ground source terminal can send cells by the reservation rate. If this request is refused, the onboard switching sends the connection setup failure message to the ground source terminal. Then the ground source terminal can't send cells. The process is shown as Fig. 1.

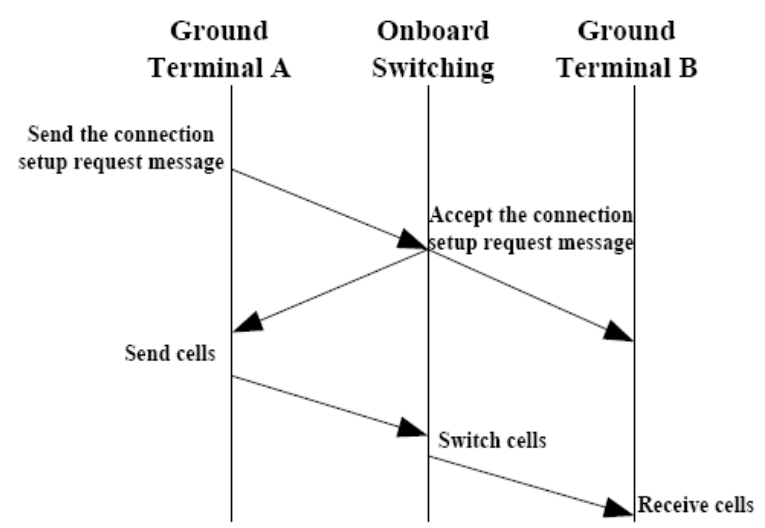

Fig. 1. When the connection is set successfully, the ground source terminal can send cells

Secondly, when the congestion will appear in the onboard switching on hand, the onboard switching should send alert message to the ground source terminals according to the number of connection and cells in the onboard buffer. Fig. 2 shows this process. After receiving the alert message, the ground terminal should stop to send cells until the congestion is mitigated in the onboard switching.

$\begin{array}{ccc}\text { Ground } & \text { Onboard } & \text { Ground Net } \\ \text { Terminal A } & \text { Switching } & \text { Control Center }\end{array}$

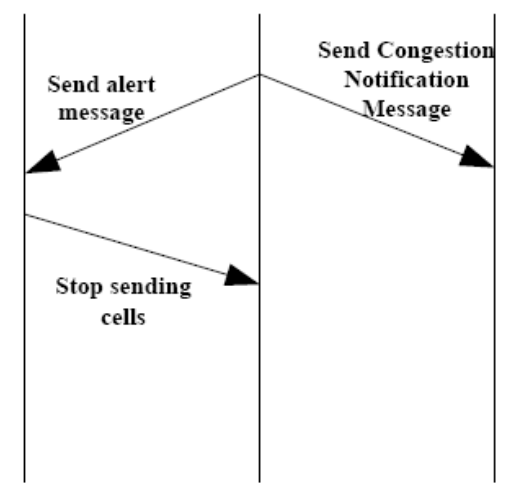

Fig. 2. When the congestion will appear on hand, the onboard switching sends alert message to ground terminal.

Because the onboard switching has get the parameters of the connection, such as services metric, traffic type, QoS requirement, and so on the switching encapsulates these information as the label of the inner cells. When the number of cells in the onboard buffer arrives at the pre-established alarm thresholds the onboard switching sends alert message by inner label to ground source terminals which send disobedient cells or affect the flow of onboard switching extremely. At the same time, this information is reported to the ground network control center. The ground source terminals which receive the alert message should stop sending cells because of the congestion in the onboard switching. This state will stand for a period of time, and then the ground source terminal resumes the normal sending state.

After sending the alert message by onboard switching, if the congestion isn't mitigated and the number of cells in the onboard buffer arrives at the pre-established discard thresholds, the onboard switching should discard some cells.

\section{B. Discarding Strategy}

The discarding strategy in this paper adopts the way of connection oriented. The identifier in the head of the cells can guarantee that the discarded cells belong to one connection, and the discarded connection can be also chosen according to this identifier. When setting up one connection, the parameters of services metric, traffic type and QoS requirements are identified by the onboard switching and this information are saved in the onboard forwarding table. When the cells are sent to the onboard switching, the switching encapsulates this information as the inner label of the inner cells, which can be used to discard cells.

The disobedient cells should be discarded firstly, and then the low priority cells are discarded. If the cells of this discarded connection arrive at the onboard switching, the cells are discarded continuously when the number of cells in the onboard buffer exceeds the pre-established discard thresholds; and the cells are reserved when the number of cells under the pre-established discards thresholds. At the same time, the onboard switching should send notification message to the ground network control center and the ground source terminal when sends the discarded cells.

Fig. 3 shows the process of discarding cells. In this figure, the onboard switching discards all cells in one connection, and then notifies the ground terminal. The connection information is saved in the identifier of cells, such as identifier of input and output port, priority, and so on; therefore it can be guarantee that the discarded cells belong to one connection. Then the feedback is sent to the ground source terminal when the cells are discarded.

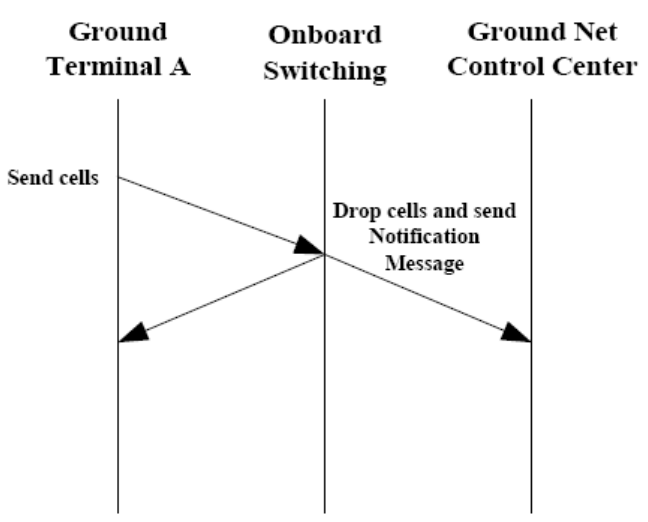

Fig. 3. After congestion, the onboard switching discards cell, and sends notification cell to the ground terminal. 


\section{Simulation}

\section{A. Simulation Model}

In this chapter, the processes of sending buffer and switching cells are simulated by Matlab. Then the performance between the algorithm of congestion control based on satellite switching and the other algorithm without congestion control strategy are compared. Because the satellite has multi-beams and every beam has one corresponding port of switching. The switching we choose to simulate and study has eight ports.

The generation, buffer, switching and output are finished in one slot at the same time and one slot means the time of switching one cell. Suppose the arrival processes of every port are mutually independent, and the buffer sizes of input and output queues are big enough. The traffic model of input port can be described as two stochastic processes, which are the process of cell generation and the process of output port selection.

The common arrival processes of cells have two kinds, which are the independent Bernoulli distribution and the burstiness process with Geometric distribution. Because the character is similar between the burstiness process and the real arrival process, the burstiness process is chosen to be simulated in this chapter.

The burstiness process can be simulated by the model of ON/OFF, in which many cells have the same destination address for every burstiness slot. Suppose the lengths of burstiness and static are Geometric distribution with the parameters are $P b$ and $P e$ respectively.

The probability distribution function of $\mathrm{ON}$ state is the probability of sending burst cells for x slots:

$$
p(x)=P b \times(1-P b)^{x-1}, x=0,1,2,3
$$

The probability distribution function of OFF state is the probability of static state standing of $\mathrm{x}$ slots:

$$
p(x)=P e \times(1-P e)^{x}, x=0,1,2
$$

In the above formulae, $\mathrm{x}$ can be zero, which means sending cells continuously. There are two different data streams, and the destination ports are different between the two streams.

The parameters in the actual emulation are average burst length $\mathrm{M}$ and the payload $\mathrm{L}$, and then we can compute that

$$
P b=1 / M, P e=1 /(M-M \times L)
$$

The simulation process is as follows:

A random number $u_{i}$ is generated before sending a cell, which is between 0 and 1.The cell burst length $i$ and the idle slots length $\mathrm{j}$ can be computed as the formulae.

$$
i=\left[\frac{\ln \left(1-u_{i}\right)}{\ln (1-P b)}\right] \quad j=\left[\frac{\ln \left(1-u_{i}\right)}{\ln \left(1-P_{e}\right)}-1\right]
$$

After computing the number $i$ and $j$, the same cell is generated in the continuous $i$ slots, that means these cells will be sent to the same output port. And then the idle cells will be generated in the latter $\mathrm{j}$ slots. Every input port is independent of others in the simulation, and the valid cells are decorrelated to the idle cells. It will appear that more than one cell are sent to the same output port, but some other output ports may be idle at the same time.

\section{B. Simulation Results}

The simulation time in this section is 100000 slots, and the arrival process of cells is the burstiness process. Then the cell loss rates are simulated and analyzed between the algorithm of congestion control based on satellite switching and the other algorithm without congestion control strategy. The performance of the proposed congestion control algorithm can be verified, and it will be found that whether the purpose of congestion control can be realized. When there are not any default cells, the satellite switching doesn't drop the cells with the congestion control algorithm proposed by this paper. Consequently the cell loss rates of the two strategies are simulated and compared only when there are some default cells.

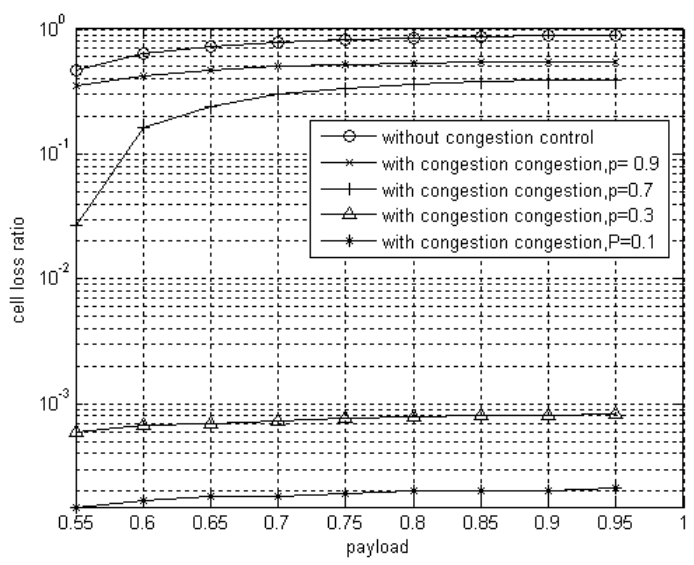

Fig. 4. Cell loss ratio between the algorithm of congestion control based on satellite switching (different probabilities for default cell) and the other algorithm without congestion control strategy.

Fig. 4 compares cell loss ratio between the algorithm of congestion control based on satellite switching and the other algorithm without congestion control strategy, for which the payload is from 0.55 to 0.95 and the burst length is 16 . In this figure, the cell loss rates of some different probabilities for default cell are also compared.

The figure shows that the proposed algorithm has the smaller cell loss ratio than the other algorithm without congestion control strategy. The conclusion is found from the simulation that the proposed algorithm in this paper, which combines the open-loop and close-loop based on the current state of onboard buffer, can reduce cell loss ratio of onboard switching obviously.

\section{CONCLUSION}

The congestion control algorithm based on satellite switching is proposed in this paper. The different strategies are adopted to control the congestion, which combine the open-loop and close-loop based on the current state of onboard buffer. This algorithm can control the congestion in order to improve satellite switching performance. At the same time, the cell loss rates are simulated and analyzed between the algorithm of congestion control based on 
satellite switching and the other algorithm without congestion control strategy, and the arrival process of cells is the burstiness process. The analysis and simulation results show that the proposed algorithm has the better performance than others in cell loss rate, which can mitigate congestion of onboard switching. Consequently, this algorithm can be adopted in the onboard switching, the resource of which is limited.

\section{ACKNOWLEDGMENT}

The research work was supported by the National Natural Science Foundation of China under Grant No.61372175 and the National Key Laboratory Foundation of China under Grant No.9140C530403130C53192.

\section{REFERENCES}

[1] H. X. Qin, Z. L. Zeng, and B. Q. Wang, "The satellite network application based on onboard switching," in Proc. the Ninth Communication Conference, March 2013, pp. 6-11.
[2] Y. D. Sun, Z. Z. Ji, and H. Wang, "Survey of congestion control for satellite networks," Telecommunications Science, pp. 66-72, Nov 2010.

[3] A. Giannoulis, T. Salonidis, and E. Knightly, "Congestion control and channel assignment in multi-radio wireless mesh network," IEEE, 2008.

[4] X. J. Xiong, "Research on congestion control algorithm for satellite link based on detection mechanism," Spacecraft Engineering, vol. 21, pp. 87-90, 2012, no. 5.

[5] J. L. Li, Y. Zhang, and J. Li, "A simulation of congestion control algorithm based on satellite IP switching," 2011 Electronics, Communications and Control (ICECC), pp. 4119-4121, 2011.

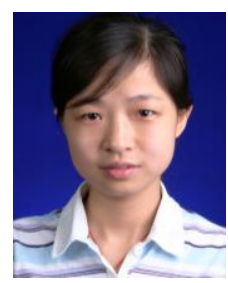

Zhang Yi is from Shaanxi province, and she was born in December 22, 1981. She was graduated from China Academy of Space Technology, and she got her master degree in satellite communication in the year of 2007.

She works on the communication signal processing and attends the national projects on network and switching in China Academy of Space Technology (Xi'an). 